\title{
Barriers and Coping Strategies of Female Teachers' Leadership Development in Primary and Secondary Schools
} An Empirical Study Based on City A in Inner Mongolia

\author{
Xiaochen Yan $^{1, *}$
}

\author{
${ }^{I}$ Ningxia University \\ *Corresponding author. Email: YXCNNX@tom.com
}

\begin{abstract}
In the context of the new era, by taking female teachers in city A of Inner Mongolia as the research subjects and adopting the interview method and NVivo qualitative analysis tool, a three-level coding analysis of the interview content shows that the obstacles of female teachers' leadership development in primary and secondary schools involve four dimensions: individual, family, school and society, such as self-trait and value orientation, family weighting and role positioning, school empowerment and work content, and gender perception and traditional culture. Besides, this study also proposes strategies to address these issues.
\end{abstract}

Keywords: female teachers, leadership development, barriers, coping strategies, primary and secondary schools

\section{INTRODUCTION}

Teacher leadership research originated from the exploration of teacher professionalization in the American education reform in the 1980s [1]. With the continuous development of education, researchers have gradually realized that the growth of schools can't only rely on the leadership of headmasters but also need to mobilize the power of teachers to participate in decision-making and leadership behavior. As female teachers account for most teachers in China, focusing on their leadership development and enhancement has become a way to improve the quality of teachers and school education.

The influence of feminist theory permeated education with the publication of Sex, Gender and Society by British scholar Ann Oakley in 1972, and female teachers had been paid increasing attention by researchers, giving rise to the research field of gender and education emerged [2]. As the women's liberation movement unfolded, women's social status gradually elevated, but influenced by the continuation of millennia of traditional culture, women teachers are still struggling in their career paths. They often play multiple roles due to their work responsibilities. Although some studies show that the leadership of women teachers is extensive, it is hindered by various factors in the absence of distraction. Besides, research shows that although women teachers have widely entered middle leadership positions in all fields, they still struggle to break through to the top. Women's leadership barriers have evolved from 'concrete walls' to 'glass ceilings' to ' labyrinths' [3]. During such historical changes, it is crucial to find the factors that constrain female teachers' leadership development, break entirely gender barriers, and encourage female teachers to participate in decision-making and leadership activities, thereby promoting the reform and innovation of educational leadership mechanisms.

\section{LITERATURE REVIEW}

Female leadership means that both the carrier of leadership and the subject of leadership activities are women [4]. Foreign research on female leadership is very fruitful. The majority of scholars believe that research in this area is a significant source of research to enhance the effectiveness of female leadership activities and an important starting point for understanding the new changes in leadership in the 21 st century. 
Some scholars have examined the impact of second-generation gender bias on women's leadership development and discussed gender discrimination in women's career development, starting with Hymowitz and Schellhardt, who first coined the term 'glass ceiling' . The glass ceiling is an invisible and, indeed, insurmountable barrier to women's efforts to lead or seek senior positions [5]. Eagly and Carli later used the term 'labyrinth' to describe the many obstacles women face in their career development, like a maze that can be seen but requires repeated navigation to find a way out. In recent years, scholars have gradually shifted from deliberately excluding women from organizations to studying invisible organizational culture and gender power inequality, proposing the

'second-generation gender bias' and constructing the theory of female leadership development [6]. There are many factors that limit the development of female leadership, one of which is the dilemma of constructing female leadership identity. Women either have to break the gender stereotype or unmotivatly ask for it or choose to give it up. In most economically underdeveloped regions, women lack the willingness and opportunities to participate in politics, which creates a mindset that women in this group find it difficult to assume responsibility, thereby leading to inertia and the fact that no one is trying to break this old balance [7]. In addition, the family remains a liability for women's leadership development, which is severely limited by the lack of networks and access to support [6].

American professors of educational administration Jonathan and Pigford in their book Women in School Leadership: A Guide to Survival mentioned that women teach and men manage schools [8], which explain the dilemma of female teachers' leadership development. The French scholar Bourdieu argues that through cultural arbitrariness, schools spread the culture of the dominant class and belittle or weaken the culture of other courses. This argument also coincides with the nature and purpose of education, highlighting the inequality within early education and the declining of female teachers community under a patriarchal social system. The subsequent female deprivation theory attributes women's inability to achieve the same academic power as men to biological and psychological factors that prevent them from devoting their time and energy, which treating female barriers as factors that promote the development of female teachers' leadership, blinding the public to the alienation of female teachers' role [9].

In the research paradigm of female teachers in the United States, the study object is mostly the selection of female experience and the construction of multiple identities of outstanding female teachers, resulting in female empiricism that the dominant position of female teachers in the field of education is superficial, a phenomenon that forms a new form of 'obscuration' of women in education. Research shows that the lower level of schooling, the greater the number of female teachers are, and their participation in decision-making and leadership is disproportionate to their large numbers [2]. A study conducted in 2004 on the Campus of Case Western Reserve University showed that female teachers generally felt they received less support and respect from the organization than their male counterparts, and were often treated differently because of their gender, they experienced more pressure and restrictions [5].

A survey on the current situation of teachers' gender consciousness in China found that teachers' level of gender consciousness in the stage of primary education is not high, mainly manifested in the immature view of gender equality and the work-family conflict of female teachers is more prominent. The traditional gender concept dramatically influences their development concept, and there are gender stereotypes [10]. Rosenthal (1976) argued that work-family conflict (WFC) results from the interdimensional interaction between work and family. Schools should genuinely care for and respect teachers, empower women teachers [7], innovate systems, use developmental teacher evaluation systems, and create a positive interpersonal environment on campus to effectively mitigate WFC. Besides, families ought to provide more understanding and support [11], and society should recognize and eliminate prejudice against female leaders and help women strengthen their social capital [6].

Compared with foreign countries, China's research on this issue is slightly inadequate. The research objects and methods are relatively single, with regional differences in research results, not nationally applicable, and lack of theoretical model construction. In future research, breakthroughs can be made in the following aspects. Firstly, it needs to strengthen the structure of theoretical models and consolidate the theoretical foundation; Secondly, it is necessary to innovate research methods and expand the scope of research continuously.

\section{RESEARCH CONTENT}

\subsection{Selection of research subjects}

This study conducted extensive research on female teachers in five schools in city A of Inner Mongolia, observing their daily work, interpersonal interaction, situational interaction, teaching performance, participation in school decision-making activities, and drafting the direction and content of the interview outline through observing the content. Fifty teachers of different ages and marital statuses were randomly selected to conduct in-depth interviews with their age and teaching subject. The interviews mainly focused on 
the interviewees' journeys, lives, and experiences, to understand their perceptions and expectations of the concepts of teacher leadership and educational leadership, and to obtain as much information as possible about their roles, value identities, self-efficacy, and professional development dilemmas and challenges. The survey also sought to acquire detailed information about roles, value identity, self-efficacy, and professional development dilemmas and challenges.

\subsection{Collection and analysis of research data}

All recordings were converted into text based on the in-depth interviews, and NVivo11 qualitative analysis software was used to encode the textual information. It was found that what the interviewees talked about could be analyzed from four dimensions: individual, family, school and society. Therefore, this research conducted open coding, spindle coding and selective coding for interview records, and specifically analyzed the four dimensions of self-traits and value orientation, family weighting and role positioning, school empowerment and work content, gender perception and traditional culture.

\section{DISCUSSION}

The above analysis found that female teachers in primary and secondary schools are generally family-oriented. Most of them are confined to traditional culture and ideas, forming a kind of habit, showing a status of working condition, believing that the appointment of leaders has no motivation for self-development. Moreover, they are more passive in general, with a more one-sided understanding of teacher leadership, believing that leadership is an ability that only leaders should have, neglecting the essence of leadership and denying the need and possibility of their leadership development. The development of female teacher leadership is faced with multiple realistic obstacles and constraints from external and internal factors.

\subsection{Gender perception and traditional culture deterring female teachers from breaking through}

The results of this study once again confirm that in some less economically developed areas, women are still clinging to gender stereotypes. $68 \%$ of the respondents believe that they can't break their habits and eventually educate their children with the traditional female role. And some female teachers with progressive ideas have stopped moving forward under the influence of conventional cultural attitudes of families or society.

\subsection{School empowerment and work content overwhelming female teachers}

It has been shown that although women are gradually moving into leadership positions, they are also concentrated in mid-level leadership positions and rarely break the shackles to reach the top. Under the leadership of male headmasters, school empowerment has become an essential constraint on the leadership development of female teachers. Gender background, work attitude and performance will affect the leadership development of female employees [12]. In the survey, $76 \%$ of female teachers reported that they were treated differently in the leadership process, such as less training and learning opportunities, being underestimated, slow promotion, and the career cycle of female teacher leaders is significantly longer than that of male teachers leaders. $83 \%$ of respondents pointed out that women's physiological characteristics and the cost of childbirth determine the physical and psychological quality of female teachers who are often physically and mentally exhausted, they find it difficult to cope with the heavy workload and the involvement of family and children, thus hindering their leadership development.

\subsection{Family weighting and role positioning weighing on female teachers}

The results of this study suggested that $81 \%$ of respondents cited family commitments as the biggest obstacle to their careers, reaffirming the findings of a survey conducted by Betz and Fitzgerald, which found that the barriers women face clashed with the pursuit of self-improvement and expectations of family responsibilities, leading women to choose lower-level or traditional jobs or even forgo better promotion opportunities. The study also found that women's family role orientation is, to some extent, determines their ability to climb the ranks. The confinement of traditional family roles hampers female leadership development, as family role orientations determine individual work goals and work schedules to a certain extent [13]. Although a gradual increase in the proportion of women in the workforce, economic independence is gradually increasing as the time move forward, men are also taking on more responsibilities in the family, women still shoulder the heavy responsibility of balancing family and work due to their biological gender characteristics and maternal ties, the social expectation of women's family role has not changed fundamentally because they participated in social work.

\subsection{Self-trait and value orientation making female teachers adapt to the current situation}

From the perspective of sociology, gender cognition affects individuals' perception, especially their 
self-efficacy, and can even influence their metacognition and value orientation. Research findings suggest that female teachers' gender perception influences their overall career development plans and achievement prediction. According to leadership theory, the development of individual leadership ability is related to one's attributes, mainly focusing on leaders' psychology, personality, knowledge, and abilities. This is in sharp contrast to male teachers' masculine and rational character, which makes female teachers generally have a low sense of self-efficacy, believe they are not suitable or incompetent for leadership, and lack of motivation and passion for work.

\section{SUGGESTION}

Based on the above-mentioned barriers to female teacher leadership development, efforts should be made by society, schools, families and individuals. To be specific, for society, it should break the traditional gender stereotype and the shackles of the feudal thought, to improve the status and influence of female teachers, reshape the orientation of gender equality, change the traditional expectation of teachers' role, abandon solidified gender symbols, foster a social environment that respects women's professional status and leadership quality of female workers, give female teachers a higher position and professional expectations, so that they can confidently present themselves. For schools, they should transform management mode and power operation mechanism, continuously improve the educational system, eliminate gender differences, give women teachers more development opportunities and promotion platforms, empower women teachers greater rights to educational leadership, innovate the way they work in education and teaching, so that female teachers can find new career directions in their busy work, conduct training and lectures related to educational leadership to inspire and train female teachers to reasonably enhance their leadership awareness and abilities, so that they can adequately deal with conflicts and contradictions between family and work. On the family side, female teachers should be given more understanding and support to reasonably play their family roles to balance family responsibilities and division of work between husband and wife, so that women can be freed from heavy domestic chores and thus have more time and energy. At the individual level, they should transcend narrow self-expectations, get rid of the shackles of traditional thinking, enhance their sense of self-efficacy by constantly challenging themselves, improve their ability to cope with life and work under pressure, carry out scientific and reasonable career planning, grasp opportunities to learn and improve, and realize their value.

\section{CONCLUSION}

Based on the evidence above, the development of female teachers' leadership is related to the professional development of the teaching force, the improvement of the quality of education and teaching in schools, and the continuous development of educational reform. Therefore, it is necessary to mobilize all parties and form a synergy to clear all obstacles on the road of female teachers' leadership development, to achieve the sustainable development of female teachers' leadership.

\section{REFERENCES}

[1] Liu Juan, The current situation of gender concept in today's society and strengthening gender awareness education, in: Journal of Hunan Radio and Television University, 2005,pp.82-84. DOI:10.3969/j.issn.1009-5152.2005.02.035

[2] Zhao Ping, On the paradigm of contemporary American teacher research in a feminist perspective, in: Comparative Education Research, 2012, pp. 67-71,76.

[3] Jiang Lai, The current situation and development trend of female leadership, in: Journal of Shanxi Normal University (Social Science Edition), 2012, pp.

123-126.

Doi:10.3969/j.issn.1001-5957.2012.04.028.

[4] Zhou Min, Characteristics of female leadership and its metaphorical meaning, in: Journal of Shanxi Normal University (Social Science Edition), 2011, pp.

Doi:10.3969/j.issn.1001-5957.2011.05.030.

[5] Sheng, Yunlong, The "glass ceiling" in female teachers' career development from the perspective of teacher structure, in: Women's Studies Series, 2009, pp. 26-31,38. Doi:10.3969/j.issn.1004-2563.2009.01.004.

[6] Xiao Wei, Luo Jinlian, Second-generation gender bias and female leadership development, in: China Human Resource Development, 2013, pp. 96-99.

[7] Zhang Lili, Zheng Xinrong, Guo Xin, Women's leadership action research: localized paths and practices, in: Journal of China Agricultural University (Social Science Edition), 2013, pp. 123-132.

[8] Pigford A B ,Tonnsen S, Women in school leadership: Survival and advancement guid book, in: Rowman \& Littlefield Education, 1993.

[9] Fang Xin, A sociological analysis of the alienation of female teachers' roles, in: Journal of Education (First Half), 2019, pp. 21-26. Doi:10.3969/j.issn.1005-3476(s).2019.08.004. 
[10] Chen Xia, Analysis of the current situation of teachers' gender awareness, Journal of Heilongjiang College of Education, 26(9) (2007) 54-56. Doi:10.3969/j.issn.1001-7836.2007.09.020.

[11] He Bing, Work-family conflicts of female teachers and their coping strategies, Journal of Economic Research, (24) 96-97. Doi:10.3969/j.issn.1673-291X.2013.24.047.

[12] Li Juan, Wang Changzhong, An analysis of the pressure and confusion of female teachers in primary and secondary schools facing promotion--a survey based on the Sino-British Southwest Basic Education Project, Teaching and Management (Theory Edition), (5) (2012) 84-85. Doi:10.3969/j.issn.1004-5872.2012.05.034.

[13] Yu Shifang, Gender bias and women's leadership development dilemma and breakthrough path, Leadership Science, (25) (2018) 54-56. 